\title{
Evaluation of The Improvement of Clinical and Radiological Scores with Doxycycline in the Treatment of Nasal Polyposis: A Randomized Double Blind Clinical Trial
}

\author{
Alireza Mohebbi ${ }^{1}$, Mohammad Aghajanpour ${ }^{1}$, Hadi Ghanbari ${ }^{1}$, Zhila Mohammadi Azar ${ }^{1}$, Ahmad Alaghehband $^{2}$ \\ \& Farshid Safdarian ${ }^{1}$ \\ ${ }^{1}$ ENT \& Head and Neck Research Center \& Department, Hazrat Rasoul Hospital, The Five Senses Institute, Iran \\ University of Medical Sciences, Tehran, Iran \\ ${ }^{2}$ Iran University of Medical Sciences, Tehran, Iran \\ Correspondence: Mohammad Aghajanpour, ENT \& Head and Neck Research Center \& Department, Hazrat \\ Rasoul Hospital, The Five Senses Institute, Iran University of Medical Sciences, Tehran, Iran. Tel: +98-91-2973- \\ 2814. E-mail: Aghajanpour560@yahoo.com
}

Received: May 29, 2019

doi:10.5539/jmbr.v9n1p142
Accepted: September 28, 2019

Online Published: October 23, 2019

\begin{abstract}
Although several trials have been proposed for nasal polyposis (NP) treatment, but no standard guideline has been declared yet. We evaluated the effect of doxycycline on NP treatment. In a double- blind randomized clinical trial, 48 patients with bilateral nasal polyposis were assigned to receive doxycycline and placebo capsules for 8 weeks and were followed monthly for 4 months. Patients were assessed for polyp size, severity of symptoms and quality of life according to the endoscopic examination, VAS score, CT scanning and SNOT-22 respectively. polyp size reduced significantly after 8 weeks of treatment in the drug group. The quality of life score has raised significantly after 2 months of treatment with doxycycline. By giving -up the drug, severity of symptoms started to grow-up after 2 months. Although a course of doxycycline improved nasal symptoms, polyp size and CT findings, but further studies with more patients and longer follow-up course are required to establish the foundation to recommend this drug as an important treatment in NP.
\end{abstract}

Keywords: Doxycycline, Nasal Polyp, Quality of Life

\section{Introduction}

Nasal polyposis (NP) has been the subject of much debate. The mounting evidence suggests that NP is a challenging clinical syndrome in which a number of possibly coexisting immunologic pathways play a role (Settipane \& Chafee, 1997). As this entity likely reflects an assay of disease states, the epidemiology is difficult to characterize (Benninger et al., 2003). While the presentation of polyps in the nasal cavity is axiomatic to the definition of chronic inflammation, the underlying etiologies are much more varied. Inflammation occurs when the activated macrophages release inflammatory mediators that stimulate burst of the plaque, resulting in narrowing of blood vessels and thrombosis (Ayaz, 2017). Recently, inflammation has been defined as "the succession of changes which occurs in a living tissue when it is injured provided that the injury is not of such a degree as to at once destroy its structure and vitality" or "the reaction to injury of the living microcirculation and related tissues" (Hemashree \& Thangavelu, 2018). Various factors may have a role in induction of inflammation (Sharaf, El-Halwagy, \& Alnahdi, 2018; Hamza \& AlSolami, 2018). Inflammation usually results from frequent oxidative stress (Al-Bishri, Hamza, \& Farran, 2017), but other factors include $\beta$ A, protease inhibitors, pentraxins, inflammatory cytokines, and prostaglandingenerating cyclooxy-genases (Qusti, Elsawi, Al batati, \& Shaker, 2017). It is a basis for most of the diseases (Shrihari, 2018; Qusti, 2017), and can negatively influence the key functions of organs, including organ failure (Al-Bishri, 2018). Inflammation is also a key physiologic defense mechanism against different factors (Osman \& Al-Shubailly, 2017).

There is a growing understanding that the diagnosis of nasal polyposis in and of itself is however; insufficient, so identifying the factors responsible for the individual's presentation can lead to not only directed treatment but also better outcomes (Bachert et al., 2003). A single final common pathway in the etiology of NP is yet to be elucidated. Leukotrienes are thought to be important in the progression of T-helper2 (Th2) mediated inflammation, 
eosinophilia and polyposis (European Aademy of Allergology and Clinical Immunology, 2007). Systemic atopy, defects of leukotriene metabolism, immune response to fungi and bacterial super-antigens have been identified that may contribute to the development of nasal polyposis (Tos \& Mogensen, 1997). An area of active investigation involves the role of immunologic responses to staphylococcus aureus enterotoxins in the pathophysiology of chronic rhinosinusitis with or without polyposis.

These proteins appear to have the ability to function as super-antigens resulting in vigorous production of Th2 cytokines including IL4, IL5, IL 13 and exotoxin, predisposing patients to nasal polyp formation (Bachert et al., 1998).

The most effective management of NP has not been clarified yet; however, medical therapy has come to play an increased role in the treatment of nasal polyposis (Herz et al., 1999).

Despite nasal polyposis being largely an inflammatory condition, infectious sinusitis may be an associated problem. Antibiotics can play an important role in treating the infectious portion of the disease. However; doxycycline and macrolide antibiotics have been used in the treatment of NP for their intrinsic anti-inflammatory properties. Treatment duration is often larger than for infectious indications, even up to one year (Lee, Rhee, \& Ming, 1998). The purpose of our study was to assess the clinical and radiological outcomes of long term low-dose doxycycline in difficult to treat chronic rhinosinusitis with polyposis.

\section{Materials and Methods}

A randomized double blind clinical trial with control group was performed from October 2017 to march 2018 in a referral university hospital (i.e. Hazrat Rasoul - Akram Hospital, Tehran, Iran).

The proposal of the research project was set forth in the ENT and Head and Neck research center and department of Hazrat Rasoul Akram Hospital, the five senses institute of Iran university of medical scinces (IUMS). The trial was undertaken in compliance with Good Clinical Practice guidelines and the ethics principles set out in the Declaration of Helsinki and was approved by the institutional research Ethics Committee.

Forty-eight patients with sinonasal polyposis over the age of 18 years old who participated to the trial were informed about objectives of the study, drug benefits and complications. All patients provided written informed consent and were asked to fill in a questionnaire in which they rated their overall symptoms on a Visual Analogue Scale $(V A S)$, on a $10 \mathrm{~cm}$ vertical line patients rated their symptoms ranging from 0 "no symptoms" to 10 "the most severe condition". In the screening procedure the patients underwent a thorough ENT clinical exam with special attention to the nasal area. The nasal cavity was explored via anterior rhinoscopy ad nasal endoscopy. Nasal polyposis was defined as the presence of visible bilateral polyps. Polyp size was determined according to the Hadley clinical scoring system (Table 1). only patients with score 3 or more, were eligible to participate. Patients were included only if none of the exclusion criteria were present (Table 2). For all patients, the epidemiologic data including the age, sex, drug intolerance and possible side effects were collected.

Table1. Polyp scoring system

\begin{tabular}{ll}
\hline Polyp score & Polyp size \\
\hline 0 & no polyps \\
1 & Small polyps in the middle meatus not reaching below middle concha \\
2 & Polyps reaching below the lower border of middle concha \\
3 & Large polyps reaching the inferior border of the inferior turbinate \\
4 & Large polyps causing complete congestion obstruction \\
\hline
\end{tabular}

After primary assessment, patients received doxycycline 100mg twice a day in the case group and placebo in the control group for 8 weeks. The pre-treatment CT-scan of all patients were graded based on Lund-Mackay scoring system which considers sinus mucosal thickening, extent of sinus opacification and obstruction of osteomeatal complex and a total score of 0 to 24 can be obtained (Hopkins, Brown, Slack, Lund, \& Brown, 2007). 
Table 2. Exclusion criteria

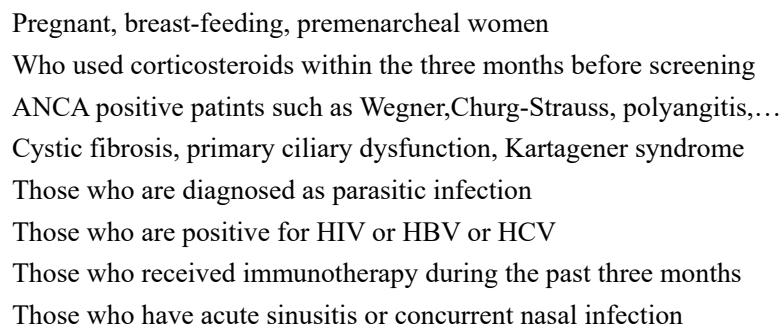

The participants' quality of life was evaluated based on the persian version of sinonasal outcome test-22 (SNOT22); consisted of 22 questions/symptoms that the patient can sort from zero (no problem) to five (worst problem possible). The patients were advised to answer the questions based on their symptoms in the two previous weeks. The sum of the values can range from 110 points and ,the higher this value, the more symptomatic the patient (Jalessi et al., 2013). All patients were monitored monthly for their compliance and possible complications. The patients' subjective scores (VAS) and endoscopic nasal polyp staging were evaluated monthly; but CT scan scoring and the patients' quality of life (based on SNOT-22) were assessed every two months.

For dealing with inter-observation variability, assessing before and after treatment was performed by one otorhinolaryngologist. We followed the patients for two months after the treatment course.

\subsection{Randomization and Study Scheme}

Eligible subjects were randomly assigned to two groups based on block design. both groups were administered $200 \mathrm{mg}$ of doxycycline and placebo capsules respectively. The capsules were unlabeled and similar to each other. Follow-up visits were scheduled for 4, 8, 12 and 16 weeks after initiating the treatment. Study personnel and participants were blind for the duration of the study; randomization codes were revealed to researchers after recruitment, data collection, and data entry.

\subsection{Statistical Analysis}

Statistical analysis was performed using SPSS version 19 software program. The student t-test was used to compare baseline values between groups. The chi-square method was used to compare qualitative variables between groups. Pairwise treatment comparisons were obtained from a 2-way ANOVA analysis. Changes in clinical and radiological parameters were evaluated by repeated measures ANOVA analyses. Differences were considered to be statistically significant if $\mathrm{p}$ - values were less than 0.05 .

\section{Results}

Forty-eight subjects met the study eligibility criteria and were randomly assigned to the drug and placebo groups. The patients' ages ranged from 18 to 72 years, with a mean of 42.29 years. None of the patients in the groups given doxycycline or placebo left the study. Only two patients in the doxycycline group reported side effects (heart burn) during the use of medication; however, there was no need to discontinue the treatment. The mean total nasal polyp scores were 6.5 and 6.58 in the doxycycline and placebo groups respectively. Overall clinical characteristics of the two groups did not differ significantly at baseline. Although Based on the mean of subjective visual analogue scale (VAS) scores, the severity of all nasal polyp symptom manifestations decreased in the drug group, however; the difference between before and after treatment in both drug and placebo groups was not statistically significant $(p-$ value $>0.05$ Figure 1).

There was a significant difference in alteration of the nasal polyp size in the drug group $(\mathrm{p}<0.02)$ in comparison with the placebo group $(\mathrm{p}>0.05)$. In 16 patients $(66.6 \%)$ in the drug group, a clear decrease in polyp size occurred while 8 patients did not show any improvements but in none of these 8 subjects the size of polyps increased. A polyp free nasal cavity was not seen in any of our cases. Although complete clearance of polyps using doxycycline was not achieved in any patient, $66.6 \%$ of patients showed an objective improvement.

The SNOT-22 score of all patients in the drug group decreased by a mean of 17.5 points, considered as significant statistically ( $p<0.01$ Figure 2, Table 3). 


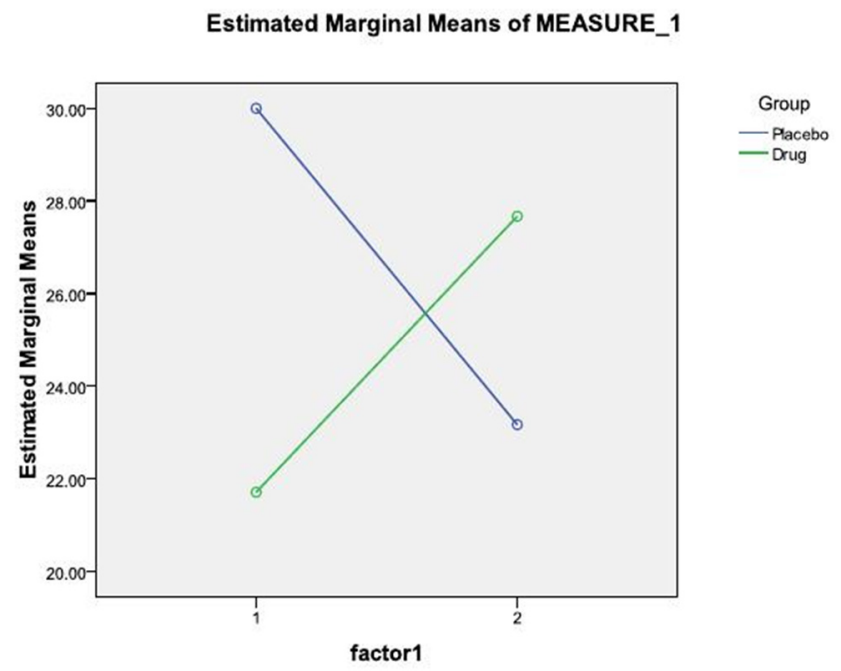

Figure 1. Alteration in severity of clinical symptoms based on VAS

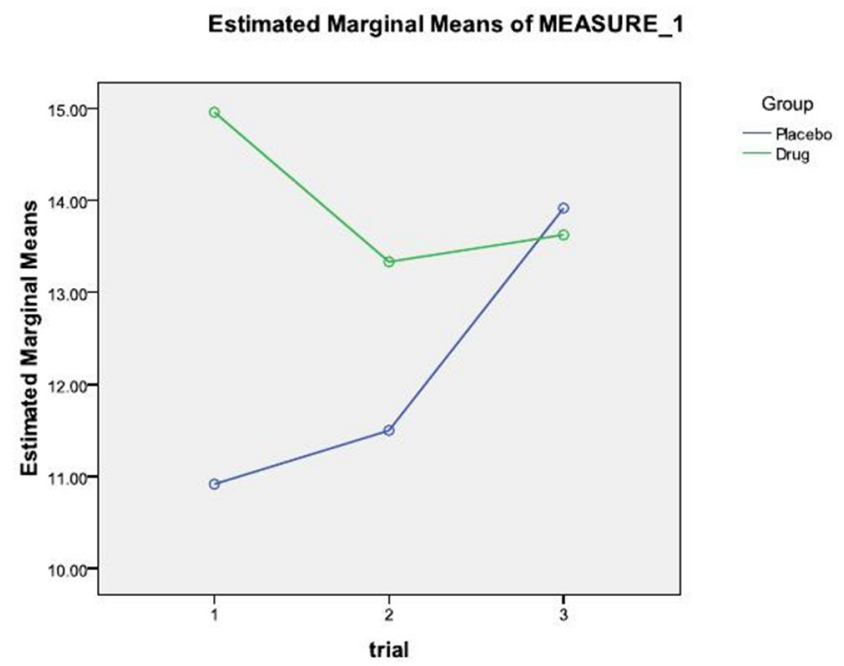

Figure 2. Alterations in patients, quality of life according to SNOT-22

Mean of Lund-Mackay score before treatment was 14.95 in the drug group which changed to 13.33 after the end of the treatment. This improvement was statistically significant $(p<0.01$ Figure 3$)$; however, the average score remained high, and a clear CT scan was not seen in any patient. Two months after completion of treatment, patients underwent endoscopic examination and paranasal sinus CT scanning again. Among 16 patients who showed decreasing polyp size after the end of two months treatment, 4 (25\%) showed signs of polyp regrowth within two months; other subjects remained stable over the follow-up period; other subjects remained stable over the followup period.

Table 3. Post-treatment results of SNOT-22

\begin{tabular}{lccc}
\hline $\begin{array}{l}\text { Group } \\
\text { CI 95\% }\end{array}$ & $\mathrm{n}$ & mean reduction of SNOT-22 & significant reduction $(>14)$ \\
\hline $\begin{array}{l}\text { Drug } \\
\begin{array}{l}15.9-53.1 \% \\
\text { Placebo } \\
49.1-80.2 \%\end{array}\end{array}$ & 24 & 17.5 & $14(58.4 \%)$ \\
\hline
\end{tabular}

Significant difference $(\mathrm{p}=0.002)$ between percentage of groups. 


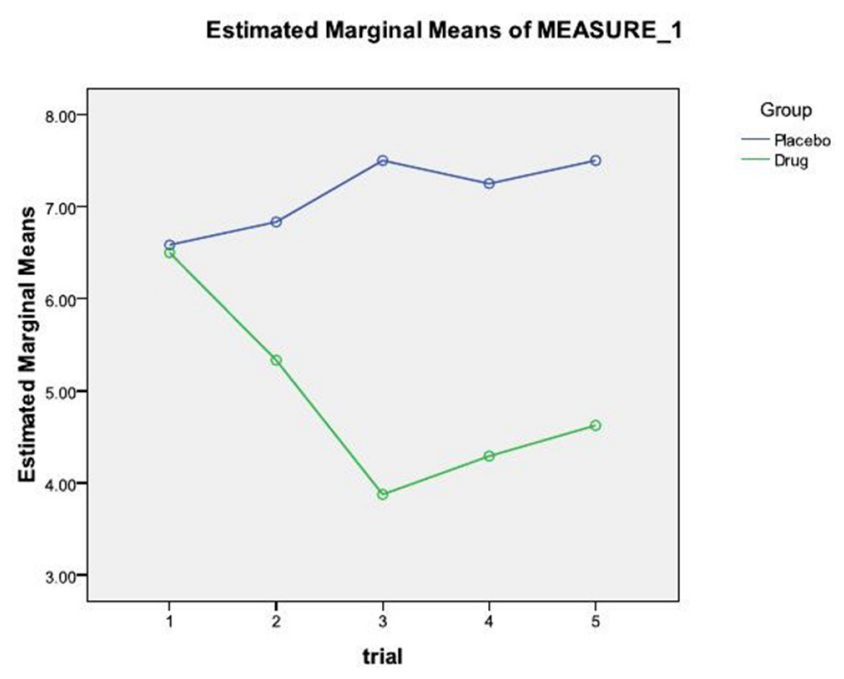

Figure 3. Alterations in Lund-Mackay score during the study

\section{Discussion}

Nasal polyposis is the most incapacitating benign disease of the nose, and its treatment is a subject of debate among clinicians.

We investigated whether the antibiotic doxycycline could decrease nasal polyp size and improve symptoms in severe nasal polyposis.

Administration of $200 \mathrm{mg}$ daily doxycycline for 8 weeks, significantly reduced polyp size which was maintained to the end of the follow-up period (week 16). This reduction in polyp size is similar to that observed after 4weeks of therapy with mometasone furoate (Small et al., 2005).

The anti-inflammatory action of doxycycline is thought to be caused by inhibition of matrix metalloprotease (MMP) (Rempe, Hayden, Robbins, \& Hoyt, 2007). In nasal polyps the ratio of MMP to its inhibitor (tissue inhibitor of metalloproteinase) is higher than in normal tissues, leading to matrix degradation, edema and chronic inflammation (Watelet, Bachert, Clayes, \& Van Cauwenberge, 2004). Doxycycline decreases allergen-induced airway inflammation and inhibits the development of bronchial remodeling by down-regulating production of IL-5 and IL-13 and activity of MMP (Gueders et al., 2008). On the other hand, doxycycline inhibits production of immunoglobulin by reduction of terminal B-cell differentiation leading to low levels of Ig E in nasal secretions (Kuzin et al., 2001).

As the purpose of our study was to determine the efficacy of doxycycline in nasal polyposis, it was necessary to find methods to evaluate the different aspects of the disease, reliably. Sinonasal outcome test-22 (SNOT-22) has been shown to be the best available test for subjective classification (Morley \& Sharp, 2006), which we consumed this method in our study. On the other hand simpler methods like VAS scoring has been used in our trial (Toros et al., 2007).

The main goal of nasal polyposis treatment is the relief of patient's symptoms and prevention of disease recurrence (Bachert, Watelet, Gevaert, \& Van Cauwenberge, 2005). The size of polyps in nasal endoscopy clearly decreased after two months of treatment; however, nasal polyps remained in all patients according to the Lund-Mackay scale.

Van Zele T. reported that doxycycline reduced the nasal polyp size significantly in the first month, prescribed for 20 days; and this reduction remained constant until the end of the third month after initiation of the drug (Van Zele et al., 2010). In our trial, CT scan scores showed significant differences in pre-treatment, 2months and 4 months following the treatment between the the two groups. Patients' quality of life did not show statistical differences in the beginning of the treatment in both groups but the differences were significant in two months following the treatment ( $\mathrm{p}$-value $=0.003$ ). Our study showed that sinus opacification improvement after treatment with doxycycline was statistically significant, but the Lund-Mackay score still remained high. This provides additional evidence that findings in CT scan do not necessarily correlate with the severity of nasal symptoms because most patients had only mild symptoms after treatment. Recent studies have shown that Lund-Mackay scoring system measures a different aspect of chronic sinusitis comparing to subjective scoring systems and the extent of the 
disease in CT scan does not correlate strongly with the severity of symptoms (Hopkins, Brown, Slack, Lund, \& Brown, 2007). Posterior nasal drip, anosmia and nasal blockage are the most disturbing symptoms in patients suffering from extensive nasal polyposis; in our study these symptoms improved significantly after treatment. As we measured these symptoms subjectively, more accurate objective tests are needed to determine the exact effect of doxycycline on PND, olfaction and nasal passage.

This study highlights the need for long-term studies with doxycycline to establish their therapeutic role in the treatment of nasal polyposis. Although four months follow-up showed favorable results but further investigations are needed. Furthermore, long term treatment with doxycycline may induce bacterial resistance which may limit this approach. Thus, supplementary controlled studies with more patients and longer follow-up should be performed in order to recommend doxycycline as a general treatment for nasal polyposis; until that time doxycycline should be considered as an adjunct treatment in extensive nasal polyposis.

\section{Conclusion}

Daily use of 200mg doxycycline for 8weeks in the population studied, showed significant improvement in quality of life as assessed by SNOT-22 questionnaire. Evaluation in the end of the fourth month of follow-up declared increasing the nasal polyp size comparing to the second month of study. It recommends that higher dosage of doxycycline or may be loner course of treatment and even adding other choices of drugs may have more effective results.

\section{Conflict of interests}

The authors declare that there is no conflict of interests regarding the publication of this paper.

\section{References}

Al-Bishri, W. M. (2018). Toxicity study of gold and silver nanoparticles on experimental animals. Pharmacophore, 9(1), 48-55.

Al-Bishri, W. M., Hamza, A. H., \& Farran, S. K. (2017). Resveratrol Treatment Attenuates Amyloid Beta, Tau Protein and Markers of Oxidative Stress, and Inflammation in Alzheimer's disease Rat Model. International Journal of Pharmaceutical Research \& Allied Sciences, 6(3), 71-78.

Ayaz, N. O. (2017). Bovine lactoferrin ameliorates cardiac muscle damage caused by nicotine toxicity by suppressing inflammatory signaling pathway. Journal of Pharmaceutical and Phytopharmacological Research, 7(2), 18-24.

Bachert et al. (1998). The role of cytokines in infectious sinusitis and nasal polyposis. Allergy, 53(1), 2-13.

Bachert et al. (2003). An update on the diagnosis and treatment of sinusitis and nasal polyposis. Allergy, 58, 17691.

Bachert, C., Watelet, J. B., Gevaert, P., \& Van Cauwenberge, P. (2005). Pharmacological management of nasal polyposis. Drugs, 65(11), 1537-52.

Benninger et al. (2003). Adult chronic rhinosinusitis: Definitions, diagnosis, epidemiology and pathophysiology. Otolaryngol Head Neck Surg, 129L, S1-S32.

European Aademy of Allergology and Clinical Immunology. (2007). European position paper on rhinosinusitis and nasal polyps. Rhinol Suppl, 20, 1-136.

Gueders et al. (2008). A novel formulation of inhaled doxycycline reduces allergen-induced inflammation, hyperresponsiveness and remodeling by matrix metalloproteinases and cytokines modulation in a mouth model of asthma. Biochem Pharmacol, 75, 514-26.

Hamza, A., \& AlSolami, F. (2018). Antitumor activity of silver nanoparticles and alpha-lipoic acid combinations in colorectal cancer induced experimentally. Pharmacophore, 9(2), 45-51.

Hemashree, J., \& Thangavelu, L. (2018). Anti - Inflammatory action of Acacia Catechu seed extract. Journal of Advanced Pharmacy Education \& Research, 8(3), 92-95.

Herz et al. (1999). Airway exposure to bacterial superantigens (SEB) induces lymphocyte-dependent airway inflammation associated with increased airwayresponsiveness: A model for non-allergic asthma. Eur $J$ Immunol, 29(3), 1021-31.

Hopkins, C., Brown, J. P., Slack, R., Lund, V., \& Brown, P. (2007). The Lund-Mackay staging system for chronic rhinosinusitis: How is it used and what does it predict? Otolaryngol Hea Neck Surg, 137(4), 555-61. 
Jalessi et al. (2013). The reliability and validity of the Persian version of sinonasal outcome test-22 (SNOT-22) questionnaires. Iranian Red Crescent Med J, 15(5), 404-408.

Kuzin et al. (2001). Tetracyclines inhibit activated B cell function. Int Immunol, 13, 921-31.

Lee, C. H., Rhee, C. S., \& Ming, Y. G. (1998). Cytokine gene expression in nasal polyps. Ann Otol Rhinol Laryngol, 107(8), 665-70.

Morley, A. D., \& Sharp, H. R. (2006). A review of sinonasal outcome scoring system- which is best? Clin Otolaryngol, 31(2), 103-9.

Osman, N. N., \& Al-Shubailly, F. (2017). Anti-inflammatory, immune-modulatory and antioxidant effects of date fruit (Phoenix dactylifera) extract in rats treated with $\mathrm{AlCl}_{3}$. International Journal of Pharmaceutical Research \& Allied Sciences, 6(2), 255-262.

Qusti, S. Y. (2017). Selenium and Melatonin Attenuates Inflammation and Oxidative Stress in the Brain of Aged Rats with Aluminum Chloride -induced Alzheimer. International Journal of Pharmacentical Research \& Allied Sciences, 6(2), 277-289.

Qusti, S. Y., Elsawi, N., Al batati, R. S., \& Shaker, S. A. (2017). A pioneer study on the anti-inflammatory activities of copper (I)-nicotinate complex against Alzheimer disease. International Journal of Pharmaceutical Research \& Allied Sciences, 6(3), 56-70.

Rempe, S., Hayden, J. M., Robbins, R. A., \& Hoyt, J. C. (2007). Tetracyclines and pulmonary inflammation. Endocr Metab Immune Disord Drug Targets, 7, 232-6.

Settipane, G. A., \& Chafee, F. H. (1997). Nasal polyposis in asthma and rhinitis: A review of 6037 patients. $J$ Allergy Clin Immunol, 59, 17-21.

Sharaf, I. A., El-Halwagy, M. E. A., \& Alnahdi, H. S. (2018). Prophylactic effect of omega-3 fatty acid on gold nanoparticles toxicity in rat's brain. Pharmacophore, 9(1), 56-68.

Shrihari, T. G. (2018). Beta endorphins - novel holistic therapeutic approach to chronic inflammation associated cancer. International Journal of Pharmaceutical and Phytopharmacological Research, 8(5), 35-38.

Small et al. (2005). Efficacy and safety of mometasone furoate nasal spray in nasal polyposis. $J$ Allergy Clin Immunol, 116, 1275-81.

Toros et al. (2007). Comparative outcomes of endoscopic sinus surgery in patients with chronic sinusitis and nasal polyps. Eur Arch Otorhinolaryngol, 264(9), 1003-8.

Tos, M., \& Mogensen, C. (1997). Pathogenesis of nasal polyps. Rhinology, 15, 87-95.

Van Zele et al. (2010). Oral steroids and doxycycline: Two different approaches to treat nasal polyps. $J$ Allergy Clin Immunol, 125(5), 1069-1076.

Watelet, J. B., Bachert, C., Clayes, C., \& Van Cauwenberge, P. (2004). Matrix metalloproteinases MMP-7,MMP9 and their tissue inhibitor TIMP-1: Expression in chronic sinusitis vs nasal polyposis. Allergy, 59, 54-60.

\section{Copyrights}

Copyright for this article is retained by the author(s), with first publication rights granted to the journal.

This is an open-access article distributed under the terms and conditions of the Creative Commons Attribution license (http://creativecommons.org/licenses/by/4.0/). 